\title{
The Decompositions of Cost Variation
}

\author{
Bert M. Balk* \\ Rotterdam School of Management \\ Erasmus University \\ E-mail bbalk@rsm.nl \\ José L. Zofío \\ Department of Economics \\ Universidad Autónoma de Madrid \\ and \\ Erasmus Research Institute of Management \\ Erasmus University \\ E-mail jose.zofio@uam.es, jzofio@rsm.nl
}

May 7, 2019

\begin{abstract}
In this paper a number of meaningful and empirically implementable decompositions of the cost variation (in difference and ratio form) are developed. The components distinguished are price level change, technical efficiency change, allocative efficiency change, technological change, scale of activity change, and price structure change. Given data from a (balanced) panel of production units, all the necessary ingredients for the computation of the various decompositions can be obtained by using linear programming techniques. An application is provided.
\end{abstract}

Keywords: Cost variation, decomposition, efficiency change, technological change, index number theory.

JEL codes: C43, D24, O12.

*This paper is intended for presentation at the European Workshop on Efficiency and Productivity Analysis XVI, London, 10-13 June 2019. A preliminary version of the theoretical part was presented at the North American Productivity Workshop at Union College, Schenectady NY, 15-17 June 2000. 


\section{Introduction}

Though textbook theory generally introduces production units as being profit maximizers it turns out that usually they have more control over their inputs than over their outputs. Any attempt to raise output quantities or revenue (if there is a market for outputs) can founder at unexpected events, such as a sudden drop in the demand for the unit's products, changes in regulatory regime, and natural or technical disasters. (Think these days of a supplier to Boeing!) On the contrary, the input side seems to be more malleable and a cost decrease seems a management target that is easier to attain than a revenue increase.

However, suppose that over a certain span of years a production unit succeeds to decrease its cost, can one then ascribe this result entirely to the role of management? That would be too simple a conclusion as also at the input side there are factors beyond the control of management. Thus, it appears worthwhile to be able to discriminate between the various factors influencing cost variation, whether they are under management's control or not. This is the theme of the present paper.

The first task is to separate the effect of prices from the effect of quantities. As cost variation can be presented as a difference (in monetary terms) or a ratio, we must make a distinction between additive measures, called indicators, and multiplicative measures, called indices. The second task is to delve deeper into the sources of input quantity change. Again, textbook theory generally considers input quantity change as being endogenous, caused by exogenous factors such as technological change, output quantity change, or input price change. This, however, tacitly presupposes efficient behaviour by (the management of) the production unit. Accounting for possibly inefficient behaviour implies that two additional factors come into play, namely technical and allocative efficiency. Separating all these effects is possible if the researcher is equipped with quantifiable information about the technologies in which the production unit under consideration operates.

The literature provides a number of such decompositions. However, as will be shown, they are asymmetric in a sense to be specified below. The core contribution of the present paper is to provide a number of symmetric decompositions and, for the first time, to compare all the decompositions on a real-life dataset of production units.

The plan of the paper follows from this outline. After having provided the necessary definitions in Section 2, Section 3 discusses additive decompositions, Section 4 discusses multiplicative decompositions, and Section 5 some alternatives. Section 6 contains the application. Section 7 concludes.

\section{The setting}

We consider a single production unit (henceforth called firm), producing output quantities $y^{t}$ while employing input quantities $x^{t}$ at input prices $w^{t}(t=0,1)$. Generic output quantity, input quantity and input price vectors will be denoted by $y \in \Re_{+}^{M}, x \in \Re_{+}^{N}$ and $w \in \Re_{++}^{N}$ respectively. Assuming the usual regularity conditions, the period $t$ technology can be represented by the radial input distance function $D_{i}^{t}(x, y)$ or the cost function $C^{t}(w, y)$. The cost-minimizing input quantity 
vector will be denoted by $x^{t}(w, y)$, so that $C^{t}(w, y)=w \cdot x^{t}(w, y)$, where $\cdot$ denotes the inner product of two equally dimensioned vectors. Notice that $x^{t}(w, y)$ is homogeneous of degree 0 in input prices $w$, and thus depends only on relative input prices or the input price structure. ${ }^{1}$

This paper is concerned with the cost variation between periods 0 and 1 , which can be expressed ${ }^{2}$ additively as $w^{1} \cdot x^{1}-w^{0} \cdot x^{0}$, and multiplicatively as $w^{1} \cdot x^{1} / w^{0} \cdot x^{0}$.

\section{An additive decomposition}

The cost variation can be decomposed additively as

$$
w^{1} \cdot x^{1}-w^{0} \cdot x^{0}=\frac{1}{2}\left(x^{0}+x^{1}\right) \cdot\left(w^{1}-w^{0}\right)+\frac{1}{2}\left(w^{0}+w^{1}\right) \cdot\left(x^{1}-x^{0}\right),
$$

where the first term on the right-hand side is the Bennet input price indicator and the second term is the Bennet input quantity indicator (see Balk (2008) for definitions and properties). Grifell-Tatjé and Lovell (2000) proposed to decompose the vector of input quantity differences as

$$
\begin{aligned}
& x^{1}-x^{0}= \\
& \quad\left(x^{1}-\frac{x^{1}}{D_{i}^{1}\left(x^{1}, y^{1}\right)}\right)-\left(x^{0}-\frac{x^{0}}{D_{i}^{0}\left(x^{0}, y^{0}\right)}\right)+ \\
& \quad\left(\frac{x^{1}}{D_{i}^{1}\left(x^{1}, y^{1}\right)}-x^{1}\left(w^{1}, y^{1}\right)\right)-\left(\frac{x^{0}}{D_{i}^{0}\left(x^{0}, y^{0}\right)}-x^{0}\left(w^{0}, y^{0}\right)\right)+ \\
& \quad x^{1}\left(w^{1}, y^{0}\right)-x^{0}\left(w^{0}, y^{0}\right)+ \\
& \quad x^{1}\left(w^{1}, y^{1}\right)-x^{1}\left(w^{1}, y^{0}\right) .
\end{aligned}
$$

The first part on the right-hand side, expression (2), is a difference of two terms of the form $x^{t}-x^{t} / D_{i}^{t}\left(x^{t}, y^{t}\right)$. The last expression is the difference between the vector of actual input quantities and the vector of technically efficient input quantities which is obtained by radially contracting the first vector to the technological frontier. The expression $x^{t}-x^{t} / D_{i}^{t}\left(x^{t}, y^{t}\right)$ is thus a measure of technical efficiency in quantity units, and the difference of these expressions signifies technical efficiency change. Following Grifell-Tatjé and Lovell (2000), the inner product of expression (2) and $\frac{1}{2}\left(w^{0}+w^{1}\right)$ will be called the technical efficiency effect. It is a measure of technical efficiency change in monetary units.

The second part on the right-hand side, expression (3), is a difference of two terms of the form $x^{t} / D_{i}^{t}\left(x^{t}, y^{t}\right)-x^{t}\left(w^{t}, y^{t}\right)$. The last expression is the difference between the vector of technically efficient input quantities and the vector of cost minimizing input quantities. The expression $x^{t} / D_{i}^{t}\left(x^{t}, y^{t}\right)-x^{t}\left(w^{t}, y^{t}\right)$ is thus a measure of allocative efficiency in quantity units, and the difference of these expressions signifies whether

\footnotetext{
${ }^{1}$ If the cost function is continuously differentiable, then by Shephard's Lemma $x^{t}(w, y)=$ $\nabla_{w} C^{t}(w, y)$, the vector of first-order derivatives with respect to $w$.

${ }^{2}$ It is assumed that the periods are not to far apart, so that it is meaningful to compare money amounts. If not, an adjustment for general inflation might be necessary.
} 
the firm's allocative efficiency has bettered or worsened. Again following GrifellTatjé and Lovell (2000), the inner product of expression (3) and $\frac{1}{2}\left(w^{0}+w^{1}\right)$ will be called the allocative efficiency effect. It measures allocative efficiency change in monetary units.

Grifell-Tatjé and Lovell (2000) called the inner product of expression (4) and $\frac{1}{2}\left(w^{0}+w^{1}\right)$ the technological change effect, and the inner product of expression (5) and $\frac{1}{2}\left(w^{0}+w^{1}\right)$ the activity effect.

However, it is immediately clear from the functional structure that expression (4) in fact combines the effect of technological change (as represented by the difference between the cost-minimizing input quantity vectors under the two technologies, $x^{1}(w, y)$ and $\left.x^{0}(w, y)\right)$ and the effect of differing input price structures between the periods 0 and $1 .{ }^{3}$ Moreover, the combined effect is asymmetric in the sense that it conditions only on the period 0 output quantity vector $y^{0}$. This criticism also applies to the activity effect term, expression (5), which conditions on the period 1 technology (via $x^{1}(w, y)$ ) as well as the period 1 input price structure.

A similar decomposition was employed by Brea-Solís et al. (2015). ${ }^{4}$ Their technical efficiency effect was the same as above, but the remainder

$$
\frac{x^{1}}{D_{i}^{1}\left(x^{1}, y^{1}\right)}-\frac{x^{0}}{D_{i}^{0}\left(x^{0}, y^{0}\right)}
$$

was split into (alternatively defined) activity and technological change effects. However, both effects exhibited also asymmetries.

It appears that a more meaningful decomposition can be obtained by combining the last two parts, expressions (4) and (5), and decomposing the result into three symmetrical parts, as follows:

$$
\begin{aligned}
& x^{1}\left(w^{1}, y^{1}\right)-x^{0}\left(w^{0}, y^{0}\right)= \\
& \frac{1}{2}\left[x^{1}\left(w^{1}, y^{1}\right)-x^{0}\left(w^{1}, y^{1}\right)+x^{1}\left(w^{0}, y^{0}\right)-x^{0}\left(w^{0}, y^{0}\right)\right]+ \\
& \frac{1}{4}\left[x^{1}\left(w^{1}, y^{1}\right)-x^{1}\left(w^{1}, y^{0}\right)+x^{1}\left(w^{0}, y^{1}\right)-x^{1}\left(w^{0}, y^{0}\right)+\right. \\
&\left.x^{0}\left(w^{1}, y^{1}\right)-x^{0}\left(w^{1}, y^{0}\right)+x^{0}\left(w^{0}, y^{1}\right)-x^{0}\left(w^{0}, y^{0}\right)\right]+ \\
& \frac{1}{4}\left[x^{1}\left(w^{1}, y^{1}\right)-x^{1}\left(w^{0}, y^{1}\right)+x^{1}\left(w^{1}, y^{0}\right)-x^{1}\left(w^{0}, y^{0}\right)+\right. \\
&\left.x^{0}\left(w^{1}, y^{1}\right)-x^{0}\left(w^{0}, y^{1}\right)+x^{0}\left(w^{1}, y^{0}\right)-x^{0}\left(w^{0}, y^{0}\right)\right] .
\end{aligned}
$$

The first part, expression (6), when multiplied by $\frac{1}{2}\left(w^{0}+w^{1}\right)$, measures the technological change effect in monetary terms. The second part, expression (7), is the average of $x^{t}\left(w^{t^{\prime}}, y^{1}\right)-x^{t}\left(w^{t^{\prime}}, y^{0}\right)$ over the four possible combinations of $t, t^{\prime}=0,1$. It measures the activity effect (scale of operation of the firm). The third part, expression (8), similarly measures the effect of differing input price structures. One

${ }^{3}$ To overcome the combination, Grifell-Tatjé and Lovell $(2015,289)$ decomposed expression (4) as $\left(x^{1}\left(w^{1}, y^{0}\right)-x^{1}\left(w^{0}, y^{0}\right)\right)+\left(x^{1}\left(w^{0}, y^{0}\right)-x^{0}\left(w^{0}, y^{0}\right)\right)$, measuring the input substitution effect and the technological change effect, respectively.

${ }^{4}$ These authors actually considered profit variation $\left(p^{1} \cdot y^{1}-w^{1} \cdot x^{1}\right)-\left(p^{0} \cdot y^{0}-w^{0} \cdot x^{0}\right)$, where $p^{t}(t=0,1)$ are output prices. The restriction to cost variation is obvious. 
sees immediately that if there is no technological change, then the first part vanishes and the other two parts reduce to

$$
\frac{1}{2}\left[x\left(w^{1}, y^{1}\right)-x\left(w^{1}, y^{0}\right)+x\left(w^{0}, y^{1}\right)-x\left(w^{0}, y^{0}\right)\right]
$$

and

$$
\frac{1}{2}\left[x\left(w^{1}, y^{1}\right)-x\left(w^{0}, y^{1}\right)+x\left(w^{1}, y^{0}\right)-x\left(w^{0}, y^{0}\right)\right]
$$

respectively, since $x^{t}(w, y)=x(w, y)(t=0,1)$.

Thus, combining expressions (1), (2), (3), (6), (7), and (8), we have obtained an additive decomposition of the cost variation $w^{1} \cdot x^{1}-w^{0} \cdot x^{0}$ into six effects, respectively that of input prices,

$$
\frac{1}{2}\left(x^{0}+x^{1}\right) \cdot\left(w^{1}-w^{0}\right),
$$

technical efficiency,

$$
\frac{1}{2}\left(w^{0}+w^{1}\right) \cdot\left(\left(x^{1}-\frac{x^{1}}{D_{i}^{1}\left(x^{1}, y^{1}\right)}\right)-\left(x^{0}-\frac{x^{0}}{D_{i}^{0}\left(x^{0}, y^{0}\right)}\right)\right),
$$

allocative efficiency,

$$
\frac{1}{2}\left(w^{0}+w^{1}\right) \cdot\left(\left(\frac{x^{1}}{D_{i}^{1}\left(x^{1}, y^{1}\right)}-x^{1}\left(w^{1}, y^{1}\right)\right)-\left(\frac{x^{0}}{D_{i}^{0}\left(x^{0}, y^{0}\right)}-x^{0}\left(w^{0}, y^{0}\right)\right)\right),
$$

technological change,

$$
\frac{1}{4}\left(w^{0}+w^{1}\right) \cdot\left[x^{1}\left(w^{1}, y^{1}\right)-x^{0}\left(w^{1}, y^{1}\right)+x^{1}\left(w^{0}, y^{0}\right)-x^{0}\left(w^{0}, y^{0}\right)\right],
$$

activity (scale of operation),

$$
\begin{gathered}
\frac{1}{8}\left(w^{0}+w^{1}\right) \cdot\left[x^{1}\left(w^{1}, y^{1}\right)-x^{1}\left(w^{1}, y^{0}\right)+x^{1}\left(w^{0}, y^{1}\right)-x^{1}\left(w^{0}, y^{0}\right)+\right. \\
\left.x^{0}\left(w^{1}, y^{1}\right)-x^{0}\left(w^{1}, y^{0}\right)+x^{0}\left(w^{0}, y^{1}\right)-x^{0}\left(w^{0}, y^{0}\right)\right]
\end{gathered}
$$

and input price structure,

$$
\begin{gathered}
\frac{1}{8}\left(w^{0}+w^{1}\right) \cdot\left[x^{1}\left(w^{1}, y^{1}\right)-x^{1}\left(w^{0}, y^{1}\right)+x^{1}\left(w^{1}, y^{0}\right)-x^{1}\left(w^{0}, y^{0}\right)+\right. \\
\left.x^{0}\left(w^{1}, y^{1}\right)-x^{0}\left(w^{0}, y^{1}\right)+x^{0}\left(w^{1}, y^{0}\right)-x^{0}\left(w^{0}, y^{0}\right)\right] .
\end{gathered}
$$

If in both periods the firm is technically efficient then the technical efficiency effect vanishes. If in both periods the firm is allocatively efficient then the allocative efficiency effect vanishes. If in both periods the firm is cost efficient then both effects vanish. Technological progress (regress) occurs if the technological change effect is negative (positive). If the scale of operation does not change, $y^{1}=y^{0}$, then the activity effect vanishes. 
Although it seems that the input price structure effect is double-counted, as price structure is part of the prices as occurring in the input price effect, this is superficial. Input prices exert a two-fold effect on the cost variation, a direct (level) effect as measured by expression (11), and an indirect (structure) effect, running via the cost-minimizing input quantities, as measured by expression (16). If $w^{1}=\lambda w^{0}$ for some $\lambda>0$, then the input price structure effect vanishes but the input price effect itself not.

Combining expressions (12) and (13) delivers

$$
\frac{1}{2}\left(w^{0}+w^{1}\right) \cdot\left(\left(x^{1}-x^{1}\left(w^{1}, y^{1}\right)\right)-\left(x^{0}-x^{0}\left(w^{0}, y^{0}\right)\right)\right)
$$

which measures the joint effect of technical and allocative efficiency change. This, however, should not be confused with cost efficiency change as such. A natural, additive measure of cost efficiency at period $t$ is $C^{t}\left(w^{t}, y^{t}\right)-w^{t} \cdot x^{t}$, which is less than or equal to 0 , a larger magnitude indicating more efficiency. Thus cost efficiency change, going from period 0 to period 1 , is measured by $\left(C^{1}\left(w^{1}, y^{1}\right)-w^{1} \cdot x^{1}\right)-$ $\left(C^{0}\left(w^{0}, y^{0}\right)-w^{0} \cdot x^{0}\right)$. Using the cost function definition, this can be rewritten as

$$
w^{1} \cdot\left(x^{1}\left(w^{1}, y^{1}\right)-x^{1}\right)-w^{0} \cdot\left(x^{0}\left(w^{0}, y^{0}\right)-x^{0}\right) .
$$

Comparing expressions (17) and (18) we see that not only their sign differs, but also that the last expression includes the full effect of price level change between periods 0 and 1 .

\section{A multiplicative decomposition}

A multiplicative counterpart to expression (1) is provided by a decomposition in terms of Sato-Vartia price and quantity indices (see Balk (2008) for definitions and properties),

$$
\frac{w^{1} \cdot x^{1}}{w^{0} \cdot x^{0}}=\prod_{n=1}^{N}\left(w_{n}^{1} / w_{n}^{0}\right)^{\phi_{n}} \times \prod_{n=1}^{N}\left(x_{n}^{1} / x_{n}^{0}\right)^{\phi_{n}},
$$

where

$$
\begin{gathered}
\phi_{n} \equiv \frac{L M\left(s_{n}^{0}, s_{n}^{1}\right)}{\sum_{n=1}^{N} L M\left(s_{n}^{0}, s_{n}^{1}\right)}(n=1, \ldots, N), \\
s_{n}^{t} \equiv w_{n}^{t} x_{n}^{t} / w^{t} \cdot x^{t}(n=1, \ldots, N ; t=0,1),
\end{gathered}
$$

and $\operatorname{LM}(a, b)$ is the logarithmic mean. ${ }^{5}$ The quantity index can be decomposed multiplicatively as

\footnotetext{
${ }^{5}$ For any two positive real numbers $a$ and $b$, their logarithmic mean is defined by $\operatorname{LM}(a, b) \equiv$ $(a-b) / \ln (a / b)$ when $a \neq b$, and $\operatorname{LM}(a, a) \equiv a$. It has the following properties: (1) $\min (a, b) \leq$ $\operatorname{LM}(a, b) \leq \max (a, b)$; (2) $L M(a, b)$ is continuous; (3) $L M(\lambda a, \lambda b)=\lambda L M(a, b)(\lambda>0)$; (4) $\operatorname{LM}(a, b)=L M(b, a) ;(5)(a b)^{1 / 2} \leq \operatorname{LM}(a, b) \leq(a+b) / 2 ;(6) \operatorname{LM}(a, 1)$ is concave. More details in Balk (2008, 134-136).
} 


$$
\begin{aligned}
& \prod_{n=1}^{N}\left(x_{n}^{1} / x_{n}^{0}\right)^{\phi_{n}}= \\
& \quad \prod_{n=1}^{N}\left(\frac{x_{n}^{1} / x_{n}^{1}\left(w^{1}, y^{1}\right)}{x_{n}^{0} / x_{n}^{0}\left(w^{0}, y^{0}\right)}\right)^{\phi_{n}} \times \prod_{n=1}^{N}\left(\frac{x_{n}^{1}\left(w^{1}, y^{1}\right)}{x_{n}^{0}\left(w^{0}, y^{0}\right)}\right)^{\phi_{n}}= \\
& \quad \prod_{n=1}^{N}\left(\frac{\frac{x_{n}^{1}}{x_{n}^{1} / D_{i}^{1}\left(x^{1}, y^{1}\right)}}{\frac{x_{n}^{0}}{x_{n}^{0} / D_{i}^{0}\left(x^{0}, y^{0}\right)}}\right)^{\phi_{n}} \times \prod_{n=1}^{N}\left(\frac{\frac{x_{n}^{1} / D_{i}^{1}\left(x^{1}, y^{1}\right)}{x_{n}^{1}\left(w^{1}, y^{1}\right)}}{\frac{x_{n}^{0} / D_{i}^{0}\left(x^{0}, y^{0}\right)}{x_{n}^{0}\left(w^{0}, y^{0}\right)}}\right)^{\phi_{n}} \times \prod_{n=1}^{N}\left(\frac{x_{n}^{1}\left(w^{1}, y^{1}\right)}{x_{n}^{0}\left(w^{0}, y^{0}\right)}\right)^{\phi_{n}}= \\
& \quad \frac{D_{i}^{1}\left(x^{1}, y^{1}\right)}{D_{i}^{0}\left(x^{0}, y^{0}\right)} \times \prod_{n=1}^{N}\left(\frac{\frac{x_{n}^{1} / D_{i}^{1}\left(x^{1}, y^{1}\right)}{x_{n}^{1}\left(w^{1}, y^{1}\right)}}{\frac{x_{n}^{0} / D_{i}^{0}\left(x^{0}, y^{0}\right)}{x_{n}^{0}\left(w^{0}, y^{0}\right)}}\right)^{\phi_{n}} \times \prod_{n=1}^{N}\left(\frac{x_{n}^{1}\left(w^{1}, y^{1}\right)}{x_{n}^{0}\left(w^{0}, y^{0}\right)}\right)^{\phi_{n}} .
\end{aligned}
$$

The first factor in expression (23) is the technical efficiency effect and the second factor is the allocative efficiency effect. The joint effect is given by the first factor in expression (22). This should also not be confused with cost efficiency change. The well-known, multiplicative, measure of cost efficiency at period $t$ is $C^{t}\left(w^{t}, y^{t}\right) / w^{t} \cdot x^{t}$, which is less than or equal to 1 , a larger magnitude indicating more efficiency. Cost efficiency change, going from period 0 to period 1 , is measured by $\left(C^{1}\left(w^{1}, y^{1}\right) / w^{1}\right.$. $\left.x^{1}\right) /\left(C^{0}\left(w^{0}, y^{0}\right) / w^{0} \cdot x^{0}\right)$. ${ }^{6}$ Using the cost function definition, this can be rewritten as

$$
\frac{\sum_{n=1}^{N} s_{n}^{1}\left(x_{n}^{1}\left(w^{1}, y^{1}\right) / x_{n}^{1}\right)}{\sum_{n=1}^{N} s_{n}^{0}\left(x_{n}^{0}\left(w^{0}, y^{0}\right) / x_{n}^{0}\right)} .
$$

This is not only a kind of inverse of the joint technical and allocative effect in expression (22), but also includes the full effect of price level change between periods 0 and 1.

The third factor in expression (23) can be decomposed further as

$$
\begin{aligned}
\prod_{n=1}^{N} & \left(\frac{x_{n}^{1}\left(w^{1}, y^{1}\right)}{x_{n}^{0}\left(w^{0}, y^{0}\right)}\right)^{\phi_{n}}= \\
& \prod_{n=1}^{N}\left(\frac{x_{n}^{1}\left(w^{1}, y^{1}\right)}{x_{n}^{0}\left(w^{1}, y^{1}\right)} \frac{x_{n}^{1}\left(w^{0}, y^{0}\right)}{x_{n}^{0}\left(w^{0}, y^{0}\right)}\right)^{\phi_{n} / 2} \times \\
& \prod_{n=1}^{N}\left(\frac{x_{n}^{1}\left(w^{1}, y^{1}\right)}{x_{n}^{1}\left(w^{1}, y^{0}\right)} \frac{x_{n}^{1}\left(w^{0}, y^{1}\right)}{x_{n}^{1}\left(w^{0}, y^{0}\right)} \frac{x_{n}^{0}\left(w^{1}, y^{1}\right)}{x_{n}^{0}\left(w^{1}, y^{0}\right)} \frac{x_{n}^{0}\left(w^{0}, y^{1}\right)}{x_{n}^{0}\left(w^{0}, y^{0}\right)}\right)^{\phi_{n} / 4} \times \\
& \prod_{n=1}^{N}\left(\frac{x_{n}^{1}\left(w^{1}, y^{1}\right)}{x_{n}^{1}\left(w^{0}, y^{1}\right)} \frac{x_{n}^{1}\left(w^{1}, y^{0}\right)}{x_{n}^{1}\left(w^{0}, y^{0}\right)} \frac{x_{n}^{0}\left(w^{1}, y^{1}\right)}{x_{n}^{0}\left(w^{0}, y^{1}\right)} \frac{x_{n}^{0}\left(w^{1}, y^{0}\right)}{x_{n}^{0}\left(w^{0}, y^{0}\right)}\right)^{\phi_{n} / 4},
\end{aligned}
$$

which gives, respectively, the technological change, activity, and input price structure effect.

\footnotetext{
${ }^{6}$ Diewert and Fox (2018) defined unit cost efficiency change as cost efficiency change divided by an output quantity index.
} 
It is interesting to compare the decomposition provided by expressions (23) and (25)-(27) with an alternative, developed by Diewert (2014):

$$
\begin{aligned}
& \frac{w^{1} \cdot x^{1}}{w^{0} \cdot x^{0}}= \\
& \left(\frac{C^{0}\left(w^{1}, y^{0}\right)}{C^{0}\left(w^{0}, y^{0}\right)} \frac{C^{1}\left(w^{1}, y^{1}\right)}{C^{1}\left(w^{0}, y^{1}\right)}\right)^{1 / 2} \times \\
& \left(\frac{C^{1}\left(w^{1}, y^{1}\right) / w^{1} \cdot x^{1}}{C^{0}\left(w^{0}, y^{0}\right) / w^{0} \cdot x^{0}}\right)^{-1} \times \\
& \left(\frac{C^{0}\left(w^{1}, y^{0}\right)}{C^{1}\left(w^{1}, y^{0}\right)} \frac{C^{0}\left(w^{0}, y^{1}\right)}{C^{1}\left(w^{0}, y^{1}\right)}\right)^{-1 / 2} \times \\
& \left(\frac{C^{0}\left(w^{0}, y^{1}\right)}{C^{0}\left(w^{0}, y^{0}\right)} \frac{C^{1}\left(w^{1}, y^{1}\right)}{C^{1}\left(w^{1}, y^{0}\right)}\right)^{1 / 2} \cdot
\end{aligned}
$$

There are only four factors distinguished. The first factor on the right-hand side, expression (28), is a Fisher-type cost-function-based input price index ${ }^{7}$, comparable with the empirical Sato-Vartia input price index in expression (19). The second factor, expression (29), measures inverse cost efficiency change, which can be compared with the joint technical and allocative effects in expression (22). The third factor, expression (30), measures inverse technological change ${ }^{8}$, and must be compared with expression (25). Notice that Diewert's measure of technological change exhibits some asymmetry in that it conditions on $\left(w^{1}, y^{0}\right)$ and $\left(w^{0}, y^{1}\right)$ instead of $\left(w^{1}, y^{1}\right)$ and $\left(w^{0}, y^{0}\right)$. The fourth factor, expression (31), is a Fisher-type cost-function-based output quantity index, comparable with the activity effect in expression (26). ${ }^{9}$ Notice also that the cost ratio, $w^{1} \cdot x^{1} / w^{0} \cdot x^{0}$, occurs on both sides of the equality sign, which makes the right-hand side less attractive as a decomposition of the left-hand side.

Grifell-Tatjé and Lovell (2015, 283) proposed a slightly different decomposition, namely

\footnotetext{
${ }^{7}$ The properties of such an index are discussed in Balk (1998, 33-35).

${ }^{8}$ Dual input based technological change, going from period 0 to period 1 , is generically defined by $C^{0}(w, y) / C^{1}(w, y)$ (Balk 1998, 58).

${ }^{9}$ This output quantity index is not linearly homogeneous, unless the technologies exhibit constant returns to scale.
} 


$$
\begin{aligned}
& \frac{w^{1} \cdot x^{1}}{w^{0} \cdot x^{0}}= \\
& \quad\left(\frac{C^{0}\left(w^{1}, y^{0}\right)}{C^{0}\left(w^{0}, y^{0}\right)} \frac{C^{1}\left(w^{1}, y^{1}\right)}{C^{1}\left(w^{0}, y^{1}\right)}\right)^{1 / 2} \times \\
& \left(\frac{C^{1}\left(w^{1}, y^{1}\right) / w^{1} \cdot x^{1}}{C^{0}\left(w^{0}, y^{0}\right) / w^{0} \cdot x^{0}}\right)^{-1} \times \\
& \left(\frac{C^{0}\left(w^{0}, y^{0}\right)}{C^{1}\left(w^{0}, y^{0}\right)} \frac{C^{0}\left(w^{1}, y^{1}\right)}{C^{1}\left(w^{1}, y^{1}\right)}\right)^{-1 / 2} \times \\
& \left(\frac{C^{0}\left(w^{1}, y^{1}\right)}{C^{0}\left(w^{1}, y^{0}\right)} \frac{C^{1}\left(w^{0}, y^{1}\right)}{C^{1}\left(w^{0}, y^{0}\right)}\right)^{1 / 2},
\end{aligned}
$$

in which the asymmetry has been moved from the technological change component to the output quantity index. It is straightforward to derive structurally identical decompositions for the difference $w^{1} \cdot x^{1}-w^{0} \cdot x^{0}$ (Grifell-Tatjé and Lovell 2015, 282-288).

If $y^{0}=y^{1}$, then the fourth factor in these two decompositions vanishes, and we obtain the multiplicative variant of the decomposition proposed by Grifell-Tatjé and Lovell (2003). Notice that in this particular case all the factors are symmetric. ${ }^{10}$

\section{More decompositions}

The logarithmic mean can be used to devise two more decompositions relating the additive and multiplicative approach. The first starts with

$$
w^{1} \cdot x^{1}-w^{0} \cdot x^{0}=L M\left(w^{0} \cdot x^{0}, w^{1} \cdot x^{1}\right) \ln \left(\frac{w^{1} \cdot x^{1}}{w^{0} \cdot x^{0}}\right)
$$

and proceeds by applying expression (19) and subsequent expressions to the righthand side of expression (36). This leads to an alternative additive decomposition.

The second starts with the reciprocal version of expression (36),

$$
\frac{w^{1} \cdot x^{1}}{w^{0} \cdot x^{0}}=\exp \left\{\frac{w^{1} \cdot x^{1}-w^{0} \cdot x^{0}}{L M\left(w^{0} \cdot x^{0}, w^{1} \cdot x^{1}\right)}\right\}
$$

and proceeds by applying expression (1) and subsequent expressions to the numerator on the right-hand side of expression (37). This leads to an alternative multiplicative decomposition.

But this is still not the end of the story. An alternative to expression (19) is

$$
\frac{w^{1} \cdot x^{1}}{w^{0} \cdot x^{0}}=\prod_{n=1}^{N}\left(w_{n}^{1} / w_{n}^{0}\right)^{\psi_{n}} \times \prod_{n=1}^{N}\left(x_{n}^{1} / x_{n}^{0}\right)^{\psi_{n}},
$$

\footnotetext{
${ }^{10}$ The context here is not longitudinal measurement but benchmarking. To be precise, $t=1$ represents the actual situation of a firm as perceived by its managers, and $t=0$ the benchmark situation as designed by consultants.
} 
where

$$
\psi_{n} \equiv \frac{L M\left(w_{n}^{0} x_{n}^{0}, w_{n}^{1} x_{n}^{1}\right)}{L M\left(w^{0} \cdot x^{0}, w^{1} \cdot x^{1}\right)}(n=1, \ldots, N) .
$$

This is a decomposition in terms of Montgomery-Vartia price and quantity indices (see Balk (2008) for definitions and properties). The noteworthy feature here is that the weights $\psi_{n}$ do not add up to 1 .

We can now develop two additional decompositions. First, expression (38) can be decomposed in the same way as expression (19) was decomposed. All we have to do is to replace the weights $\phi_{n}$ by $\psi_{n}$. Notice that the technical efficiency effect then appears as

$$
\left(\frac{D_{i}^{1}\left(x^{1}, y^{1}\right)}{D_{i}^{0}\left(x^{0}, y^{0}\right)}\right)^{\sum_{n=1}^{N} \psi_{n}} .
$$

Second, combining expression (36) with expression (38) gives

$$
\begin{aligned}
& w^{1} \cdot x^{1}-w^{0} \cdot x^{0}= \\
& \quad \sum_{n=1}^{N} L M\left(w_{n}^{0} x_{n}^{0}, w_{n}^{1} x_{n}^{1}\right) \ln \left(w_{n}^{1} / w_{n}^{0}\right)+\sum_{n=1}^{N} L M\left(w_{n}^{0} x_{n}^{0}, w_{n}^{1} x_{n}^{1}\right) \ln \left(x_{n}^{1} / x_{n}^{0}\right) .
\end{aligned}
$$

The second factor on the right-hand side, being the input quantity effect, can then be decomposed into the by now well-known five components.

\section{An application: Taiwanese banking industry}

\subsection{DEA approach and data}

Any application of the decompositions developed in the previous sections presupposes knowledge of the period $t$ technology. Given data on a number of firms, which are supposed to share the same technology, this technology can be approximated by way of non-parametric Data Envelopment Analysis methods. In particular, given data $\left(x^{k t}, y^{k t}\right)$ for $k=1, \ldots, K^{t}$ and $t=0,1$, any input distance function value can be computed by solving the following linear programming problem

$$
\begin{aligned}
1 / D_{i}^{t}(x, y)= & \min _{z, \lambda} \lambda \text { subject to } \\
& \sum_{k^{\prime}=1}^{K^{t}} z_{k^{\prime}} x^{k^{\prime} t} \leq \lambda x, y \leq \sum_{k^{\prime}=1}^{K^{t}} z_{k^{\prime}} y^{k^{\prime} t}, \\
& z_{k^{\prime}} \geq 0\left(k^{\prime}=1, \ldots, K^{s}\right),\left[\sum_{k^{\prime}=1}^{K^{t}} z_{k^{\prime}}=1\right],
\end{aligned}
$$

and any cost minimizing input quantity vector $x^{t}(w, y)$ can be obtained as the solution to the following linear programming problem 


$$
\begin{aligned}
x^{t}(w, y)= & \arg \min _{z, x} w \cdot x \text { subject to } \\
& \sum_{k^{\prime}=1}^{K^{t}} z_{k^{\prime}} x^{k^{\prime} t} \leq x, y \leq \sum_{k^{\prime}=1}^{K^{t}} z_{k^{\prime}} y^{k^{\prime} t}, \\
& z_{k^{\prime}} \geq 0\left(k^{\prime}=1, \ldots, K^{t}\right),\left[\sum_{k^{\prime}=1}^{K^{t}} z_{k^{\prime}}=1\right] .
\end{aligned}
$$

The restriction between brackets in expressions (41) and (42) must be deleted in the case of imposing global constant returns to scale. However, given the different sizes of the production units in the example below we do not impose this. Distance function as well as cost function values can be computed for firm data contemporaneous with period $t$ or not. ${ }^{11}$

Our example uses data of a balanced panel of 31 Taiwanese banks over the period 2006-2010. A complete discussion of the statistical sources, variable specifications, and summary statistics can be found in Juo et al. (2015). ${ }^{12}$ The same data set has been used to illustrate the decompositions of total factor productivity change using quantities-only and price-based indices by Balk and Zofío (2018). Regarding the technology and interrelations between inputs and outputs, the variables reflect the intermediation approach suggested by Sealey and Lindley (1977), whereby financial institutions, through labour and capital, collect deposits from savers to produce loans and other earning assets for borrowers. The three inputs are financial funds $\left(x_{1}\right)$, labour $\left(x_{2}\right)$, and physical capital $\left(x_{3}\right)$. The output vector includes financial investments $\left(y_{1}\right)$ and loans $\left(y_{2}\right)$.

\subsection{Additive decompositions}

Table 2 presents the additive decomposition of cost variation between 2006 and 2010: $\Delta C_{A}^{06,10}=w^{10} \cdot x^{10}-w^{06} \cdot x^{06}$. Cost in the Taiwanese banking industry has generally decreased for all banks, with an average reduction of 6,459 million TWD, led by Bank \#2 with -26,957 million TWD. The Bennet decomposition, expression (1), shows that the main driver of the cost reduction is an input price decline to the tune of $-8,406$ million TWD on average, as shown by the input price indicator $\left(I P I_{B}\right)$. Unsurprisingly, such reduction of input prices results in an increase of the input quantities, as shown by the positive value of the Bennet input quantity indicator $\left(I Q I_{B}\right)$, whose average amounts to 1,946 million TWD. It is possible to learn about the sources of the cost reduction by resorting to the decompositions proposed by Grifell-Tatjé and Lovell (2000), expressions (2)-(5), and the one introduced here, expressions (2), (3), (6)-(8).

As many as nine banks are technically efficient in both periods, and therefore their technical efficiency change is zero, $T E_{A}=0$. Of these, four are also allocatively efficient, $A E_{A}=0$, implying that they minimize costs at their production scale

\footnotetext{
${ }^{11}$ For the linear programming problems in this paper the MATLAB toolbox developed by Álvarez et al. (2019) has been used.

${ }^{12}$ We are grateful to these authors for sharing the data.
} 
(output level), and therefore cannot perform better from an efficiency perspective. As for the remaining inefficient banks, most of them experience technical efficiency gains resulting in lower costs, $T E_{A}<0$. A remarkable example is Bank \#3, whose approach to the production frontier from 2006 to 2010 resulted in cost savings equal to 6,793 million TWD. On the other hand, seven banks exhibit greater technical inefficiency $T E_{A}>0$, but their associated cost increase never surpasses 1,000 million TWD. The role played by allocative (in)efficiency is equally important in monetary terms. Allocative efficiency reflects the ability of production units to anticipate the change of input prices from the base to the comparison year, and thereby demand the optimal input quantities, given their individual prices. Overall, the direction of allocative efficiency change is inconclusive: 11 banks experienced increasing cost, $A E_{A}>0$, and 15 decreasing cost, $A E_{A}<0$.

Technical and allocative efficiency effects are common to both decompositions because they compare prices, quantities and technologies of contemporaneous periods. However, the difference between the two decompositions emerges when mixed period evaluations are brought into the analysis. The positive effect of technological progress on cost reduction, measured as the (average) difference in optimal quantities between the comparison and base periods, keeping prices and output quantities constant, expression (6), is overvalued by Grifell-Tatjé and Lovell's (2000) definition, expression (4), in which input prices are updated: $T C_{G L}=-1.816$ million TWD vs. $T C_{A}=-1,587$ million TWD. The compatibility of individual results is rather low, as indicated by the Spearman correlation between both scores, $\rho\left(T C_{G L}, T C_{A}\right)$ $=0.2077$, which is not statistically significant at the usual levels. Likewise, the activity effect associated to cost increases resulting from output quantity growth is overvalued in the first decompositions, Act. $E_{G L}=4,038$ million TWD vs. Act. $E_{A}$ $=3,803$ million TWD. But in this case $\rho\left(\right.$ Act. $E_{G L}$, Act. $\left.E_{A}\right)=0.8145$, which is significant. It is also possible to see that the shift in the input price structure plays a negligible effect on cost reduction. Recall that the input price level effect is caught by $I P I_{B}$.

In general, we conclude that although technological progress contributes significantly to cost reduction, the effect of output quantity growth more than compensates those gains, thereby resulting in cost increases. This explains ultimately the positive values of the Bennet quantity index $\left(I Q I_{B}\right)$, and suggests the existence of scale inefficiencies in the Taiwanese banking industry, as confirmed by Balk and Zofío (2018, Section 4). We therefore conclude that, on average, the main drivers of cost reduction in the Taiwanese banking industry are the general decline of input prices, notable technological progress, and mild gains in technical efficiency. On the other hand, increased allocative inefficiency and scale effects work against cost reduction.

\subsection{Multiplicative decompositions}

Table 3 reports the results of the multiplicative decomposition of cost variation between 2006 and 2010, $\Delta C_{M}^{06,10}=w^{10} \cdot x^{10} / w^{06} \cdot x^{06}$. As cost in the Taiwanese banking industry has decreased over the period, the ratio is smaller than one for all banks, with an average reduction of $(13,017 / 19,477-1) \times 100=-33.2 \%$. The percentage change in cost is now led by Bank \#1 with a $-63.1 \%$ reduction. The 
Sato-Vartia decomposition, expression (19), shows that most of this reduction is due to decreased prices since the Sato-Vartia input price index $I P I_{S V}$ is equal to 0.6378 on average ( $-36.2 \%$ change). As a result of this price reduction, input quantities increased by $7.1 \%\left(I Q I_{S V}=1.0709\right)$. Notice that the results of both indices are consistent with the findings reported in the previous subsection, as they constitute the multiplicative counterpart of the additive approach represented by the Bennet indicators.

Following the decomposition set out in expressions (23) through (27), we can study the sources of the cost reduction. Since the quantity index increases over time on average, given the results of the previous subsection one expects index numbers greater than one except for technological change. This is the case for the technical efficiency effect, showing an average decrease in technical efficiency of $5.95 \%$, $T E_{S V}=1.0595$. This change is opposite to the average efficiency effect in the additive decomposition, $T E_{A}$, which contributes to the cost reduction with -398 million TWD. The allocative efficiency effect $A E_{S V}$ is also positive, signaling a worsening performance to the tune of $14.1 \%$, which is consistent with the average cost increase of 122 million TWD reported in Table 2 by $A E_{A}$. This simply shows that multiplicative and additive decompositions may lead to different conclusions regarding the drivers of cost change; that is, a consistent numerical relationship between the components of both decompositions does not exist. Only in the case of technical and allocative efficiency, the zero values in the additive approach correspond with index numbers equal to one in the multiplicative approach. This is the case of the nine technically efficient banks, of which four are also allocatively efficient, and therefore cost efficient. In the rest of the cases one may obtain conflicting results. For example, Bank \#16 ranks worst regarding technical efficiency change with $T E_{S V}=2.2748$, while from the additive perspective it shows cost savings of $T E_{A}=-130$ million TWD. This, however, is an exception. The same bank presents the second largest contribution to cost reduction from an allocative perspective $A E_{S V}=0.8670$, which is compatible with cost savings of $A E_{A}=-1,638$ million TWD, the fourth largest decline from the additive perspective. The pairwise Spearman correlations between the multiplicative and additive components can be found in Table 1.

Table 1: Spearman correlations between additive and multiplicative effects

\begin{tabular}{l|ccccc}
\hline & $T E_{S V}$ & $A E_{S V}$ & $T C_{S V}$ & $A c t . E_{S V}$ & $I P S_{S V}$ \\
\hline$T E_{A}$ & $0.8583^{* *}$ & -0.1637 & 0.0069 & $-0.4168^{*}$ & -0.0568 \\
$A E_{A}$ & $-0.4715^{* *}$ & $0.7765^{* *}$ & 0.3179 & -0.0813 & 0.1589 \\
$T C_{A}$ & 0.3012 & 0.1344 & $0.5903^{* *}$ & $-0.6222^{* *}$ & -0.2195 \\
$A c t . E_{A}$ & $-0.4445^{*}$ & -0.1772 & -0.1464 & $0.8073^{*}$ & -0.0758 \\
$I P S_{A}$ & 0.0327 & 0.3176 & 0.0400 & -0.2542 & 0.1193 \\
\hline \multicolumn{2}{l}{ Note: ${ }^{*} \mathrm{p}<0.01 ; * * \mathrm{p}<0.05$} & & &
\end{tabular}

The discrepancy between the multiplicative and additive components regarding technological change is much smaller. On average, technological change contributes to cost reduction by $-14.15 \%$ on average, $T C_{S V}=0.8585$, just as its additive counterpart shows decreasing cost, $T C_{A}=-1,587$ million TWD. Comparing these two 
components at the individual level, one confirms that technological progress (regress) in the multiplicative approach normally corresponds with cost savings (increases) in the additive approach. For instance, Bank \#14, whose technological progress is the one contributing most to cost reduction within the sample, $T C_{S V}=0.4993$, by reducing cost by half, also presents the largest additive cost reduction equal to $-12,649$ million TWD. As for the last two factors, capturing the contribution of the activity (scale) and the input price structure, the former is once again strongly against the observed reduction in costs, Act. $E_{S V} \geq 1$, while the effect of the change in input price structure is almost negligible, $I P S_{S V} \approx 1$. This corresponds again with the results of the additive decomposition.

The decomposition of cost variation by means of the cost function, as proposed by Diewert (2004) and later modified by Grifell-Tatjé and Lovell (2015), expressions (28)-(31) and (32)-(35), respectively, is presented in Table 4. As the distance function does not play a role in the analysis, cost efficiency change cannot be decomposed into its technical and allocative components comparable to Tables 2 and 3.

Cost reduction in the Taiwanese banking industry appears to be again mainly driven by the decline of input prices, as on average $I P I_{D}=0.5977$. This corresponds to the average Sato-Vartia input price index $I P I_{S V}=0.6378$, reported in Table 3. As for the sources of cost reduction, all the index numbers are remarkably similar to those following from the decomposition of the Sato-Vartia quantity index. First we observe that Diewert's (2004) cost efficiency factor can be compared to the cost efficiency effect that results from multiplying the technical and allocative effects in Table 3. Growing technical inefficiency detracts from cost reduction, although the effect following from the Sato-Vartia decomposition is half of that signaled by Diewert's factor: $C E_{D}=1.1318$ versus $T E_{S V} \times A E_{S V}=1.0595 \times 1.0141=$ 1.0744. The differences between the other factors compensate this gap, though the values are very similar. Technological change contributes to cost reduction with $-12.92 \%\left(T C_{D}=0.8708\right)$, and output quantity change increases cost by $32.38 \%$ $\left(O Q I_{D}=1.3238\right)$, the corresponding effects in the previous decomposition being $14.15 \%$ and $32.95 \%$, respectively. Also, as expected given that they simply interchange asymmetries, the alternative technological change and output quantity indices of Grifell-Tatjé and Lovell (2015) are almost identical on average and at the individual level: $T C_{G L}=0.8690$ and $O Q I_{G L}=1.3261$.

Finally, the decompositions of the additive and multiplicative forms of cost variation as presented in Section 5 are reported in Table 5. Here the additive cost reduction is decomposed in two factors, the logarithmic mean of base and comparison period cost and the logarithm of their ratio, expression (36). While the first is not subject to decomposition, the second can be decomposed by taking the logarithms of the Sato-Vartia input price and quantity indices, $\ln I P I_{S V}$ and $\ln I Q I_{S V}$. Subsequently, $\ln I Q I_{S V}$ can be further decomposed by taking the logarithms of all the factors presented in Table 3, corresponding to expressions (23) and (25)-(27). We leave the exercise to the interested reader as this transformation does not alter our findings regarding the sources of cost reduction in the Taiwanese banking industry.

As for the Montgomery-Vartia decomposition of the cost ratio, expression (38), in input price and quantity index numbers, $I P I_{M V}$ and $I Q I_{M V}$, we observe that 
they are equal to the their Sato-Vartia counterparts up to the third decimal place. Hence we conclude that in this particular empirical application, the use of the alternative sets of weights, $\phi^{n}$ or $\psi^{n}$, does not make a significant difference. For the sake of completenesses we report the Montgomery-Vartia counterpart to the Sato-Vartia decomposition of the input quantity index. Here larger differences can be found when comparing each pair of components, particularly for the allocative efficiency effect, which captures most of the difference: $A E_{S V}=1.0141$ versus $A E_{M V}=0.8901$ (their Spearman correlation is $\rho\left(A E_{S V}, A E_{M V}\right)=0.5777$, which is statistically significant). In general, however, the two decompositions are compatible. The input price structure effect shows more volatility in Table 5 than in Table 3.

The alternative decomposition in expression (40) yields input price and quantity indicators comparable to the Bennet indicators in Table 2. The outcomes are remarkably similar on average, with slight variations for individual banks; a large decrease of input prices and a mild increase in input quantities.

\section{Conclusion}

A firm's cost variation through time can be expressed by a difference as well as a ratio. The decomposition of the cost difference proposed by Grifell-Tatjé and Lovell (2000) was shown to be not completely satisfactory and could be replaced by a more meaningful one. The present paper also provided a structurally identical decomposition of the cost ratio. Using the powerful tool of the logarithmic mean, four additional decompositions could be developed, two for the cost difference and two for the cost ratio. All in all, the cost variation can be decomposed in at least six structurally identical, but empirically different ways. It remains to be seen whether there are criteria for choosing between them.

Given data from a (balanced) panel of firms, all the necessary ingredients for the computation of the various decompositions can be obtained by using linear programming techniques. In this paper a dataset of 31 Taiwanese banks over the years 2006-2010 has been used to illustrate the empirical differences between the various decompositions.

It appeared that on average the additive Bennet price and quantity indicators correspond closely to their multiplicative Sato-Vartia and Montgomery-Vartia counterparts. All these measures signal that the overall cost reduction in the industry was driven by input prices, partially offset by input quantities. Also, once the input quantity components were decomposed so as to learn about the deeper lying components of cost reduction, at the level of individual production units additive and multiplicative measures may yield different results, notably regarding the direction of technological change and the activity effect. All in all, this paper makes a case for preferring symmetric to asymmetric decompositions.

\section{References}

[1] Álvarez, I., J. Barbero and J. L. Zofío, 2019,"A Data Envelopment Analysis Toolbox for MATLAB", Journal of Statistical Software, to appear. Available from 
http://www.deatoolbox.com.

[2] Balk, B. M., 1998, Industrial Price, Quantity, and Productivity Indices: The Micro-Economic Theory and an Application (Kluwer Academic Publishers, Boston / Dordrecht / London).

[3] Balk, B. M., 2008, Price and Quantity Index Numbers: Models for Measuring Aggregate Change and Difference (Cambridge University Press, New York).

[4] Balk B. M. and J. L. Zofío, 2018, The Many Decompositions of Total Factor Productivity Change. ERIM Report Series Research in Management, No. ERS2018-003-LIS (Erasmus Research Institute of Management, Erasmus University, Rotterdam). Available from http://hdl.handle.net/1765/104721.

[5] Brea-Solís, H., R. Casadesus-Masanell and E. Grifell-Tatjé, 2015, "Business Model Evaluation: Quantifying Walmart's Sources of Advantage", Strategic Entrepreneurship Journal 9, 12-33.

[6] Diewert, W. E., 2014, "Decompositions of Profitability Change Using Cost Functions", Journal of Econometrics 183, 58-66.

[7] Diewert, W. E. and K. J. Fox, 2018, "A Decomposition of US Business Sector TFP Growth into Technical Progress and Cost Efficiency Components", Journal of Productivity Analysis 50, 71-84.

[8] Grifell-Tatjé, E. and C. A. K. Lovell, 2000, "Cost and Productivity", Managerial and Decision Economics 21, 19-30.

[9] Grifell-Tatjé, E. and C. A. K. Lovell, 2003, "The Managers versus the Consultants", Scandinavian Journal of Economics 105, 119-138.

[10] Grifell-Tatjé, E. and C. A. K. Lovell, 2015, Productivity Accounting: The Economics of Business Performance (Cambridge University Press, New York).

[11] Juo, J.-C., T.-T. Fu, M.-M. Yu and Y.-H. Lin, 2015, "Profit-Oriented Productivity Change", Omega 57, 176-187.

[12] Sealey, C. W. and J. T. Lindley, 1977, "Inputs, Outputs, and a Theory of Production and Cost at Depository Financial Institutions", The Journal of Finance $32,1251-1266$. 


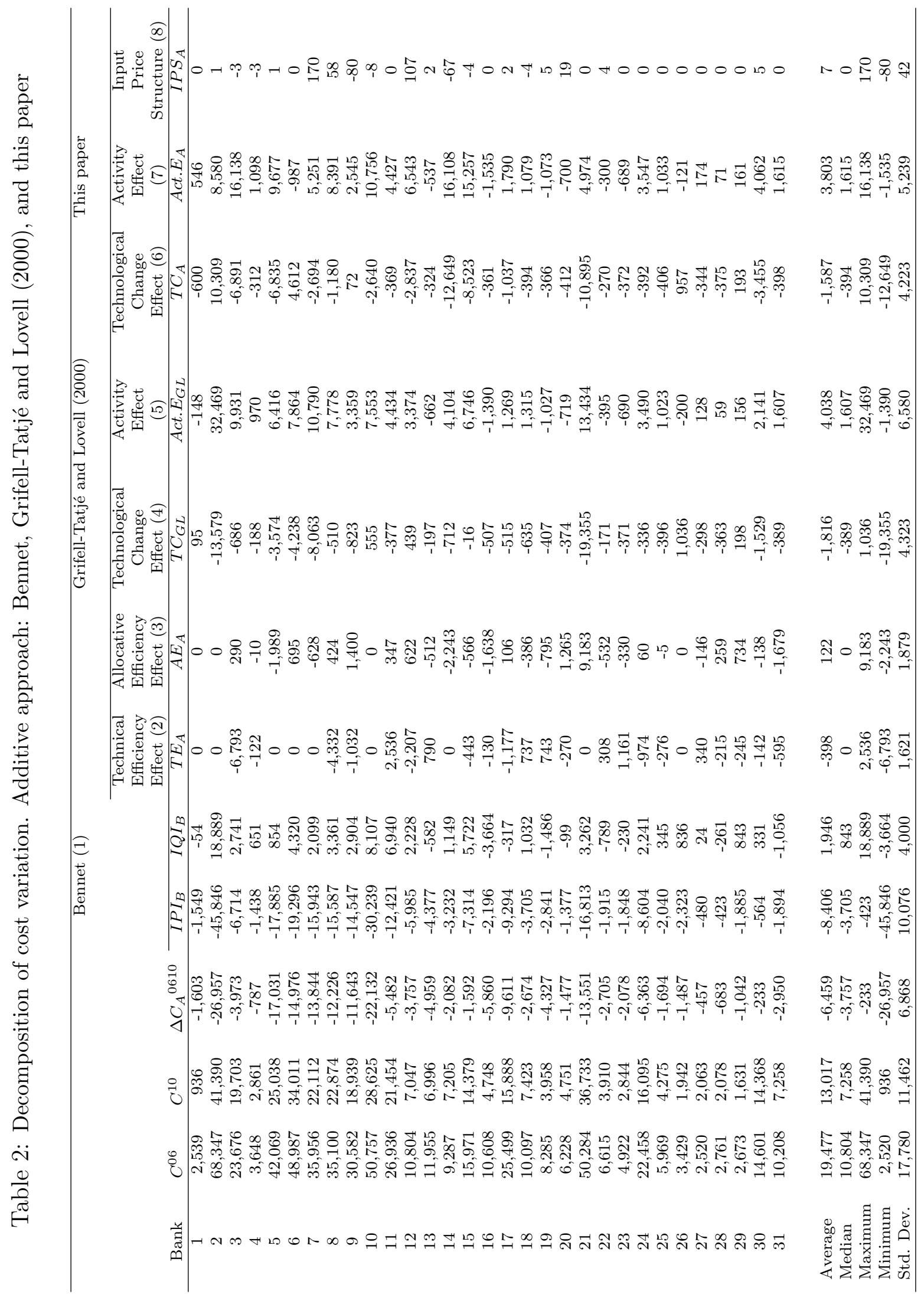




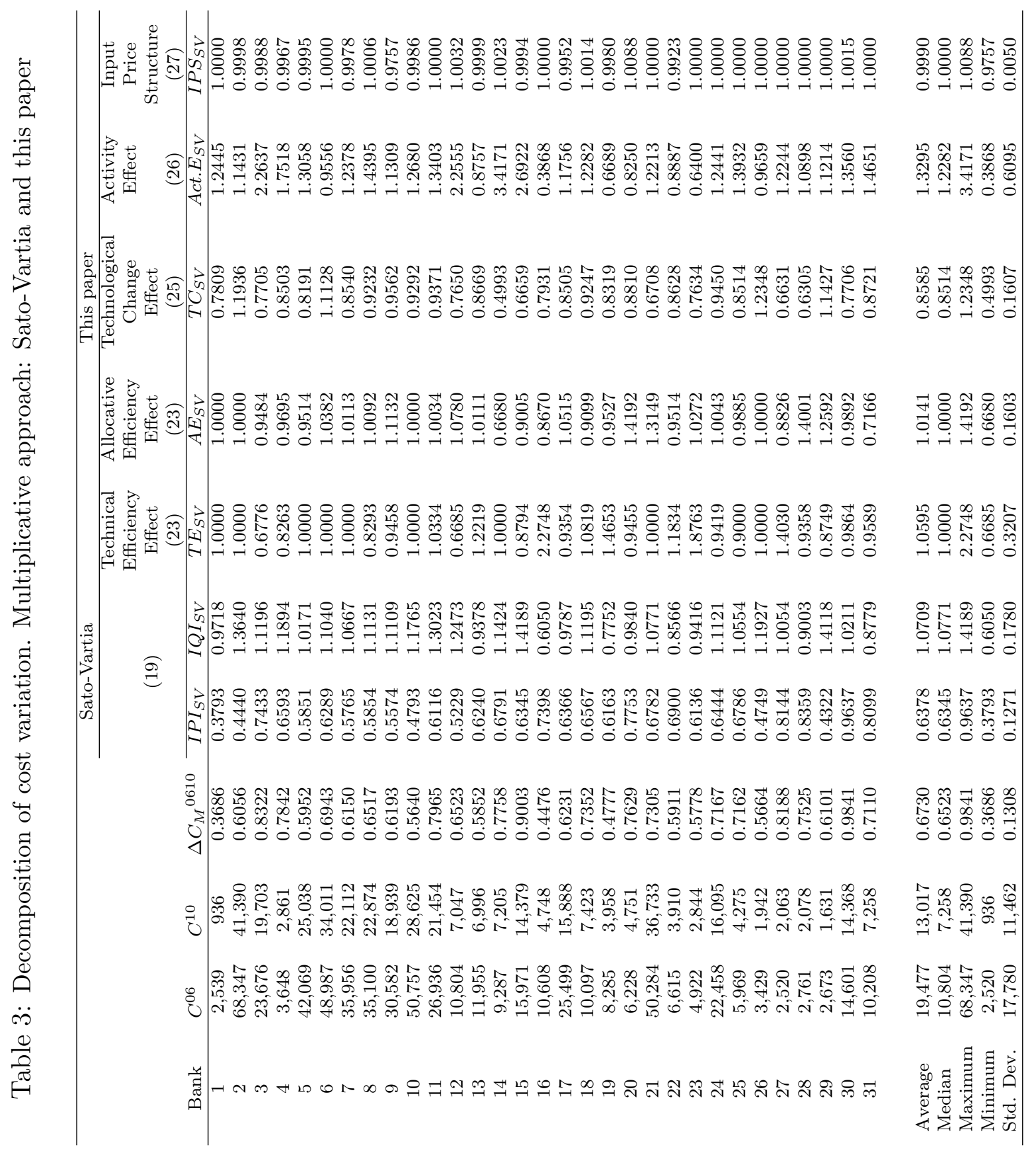




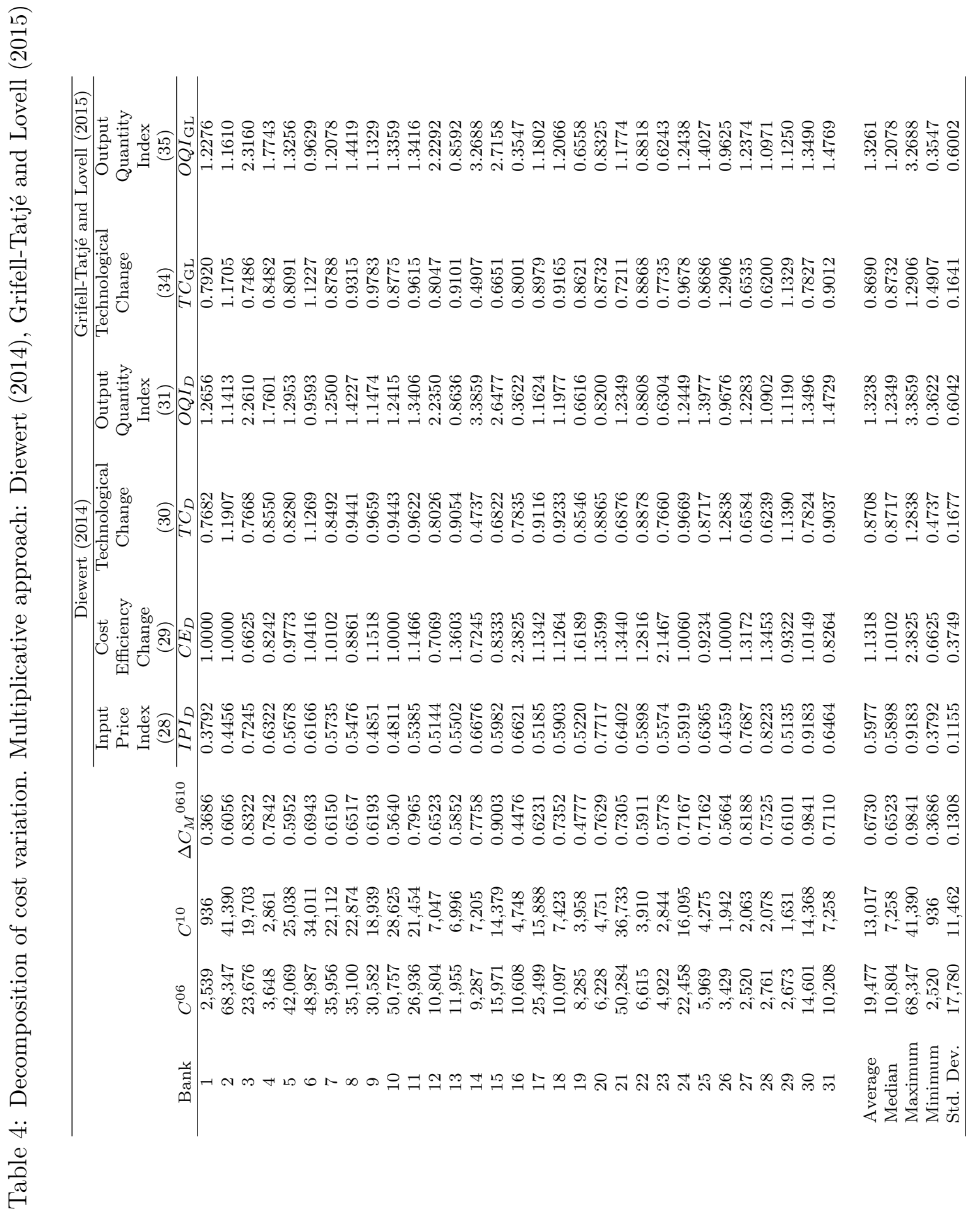




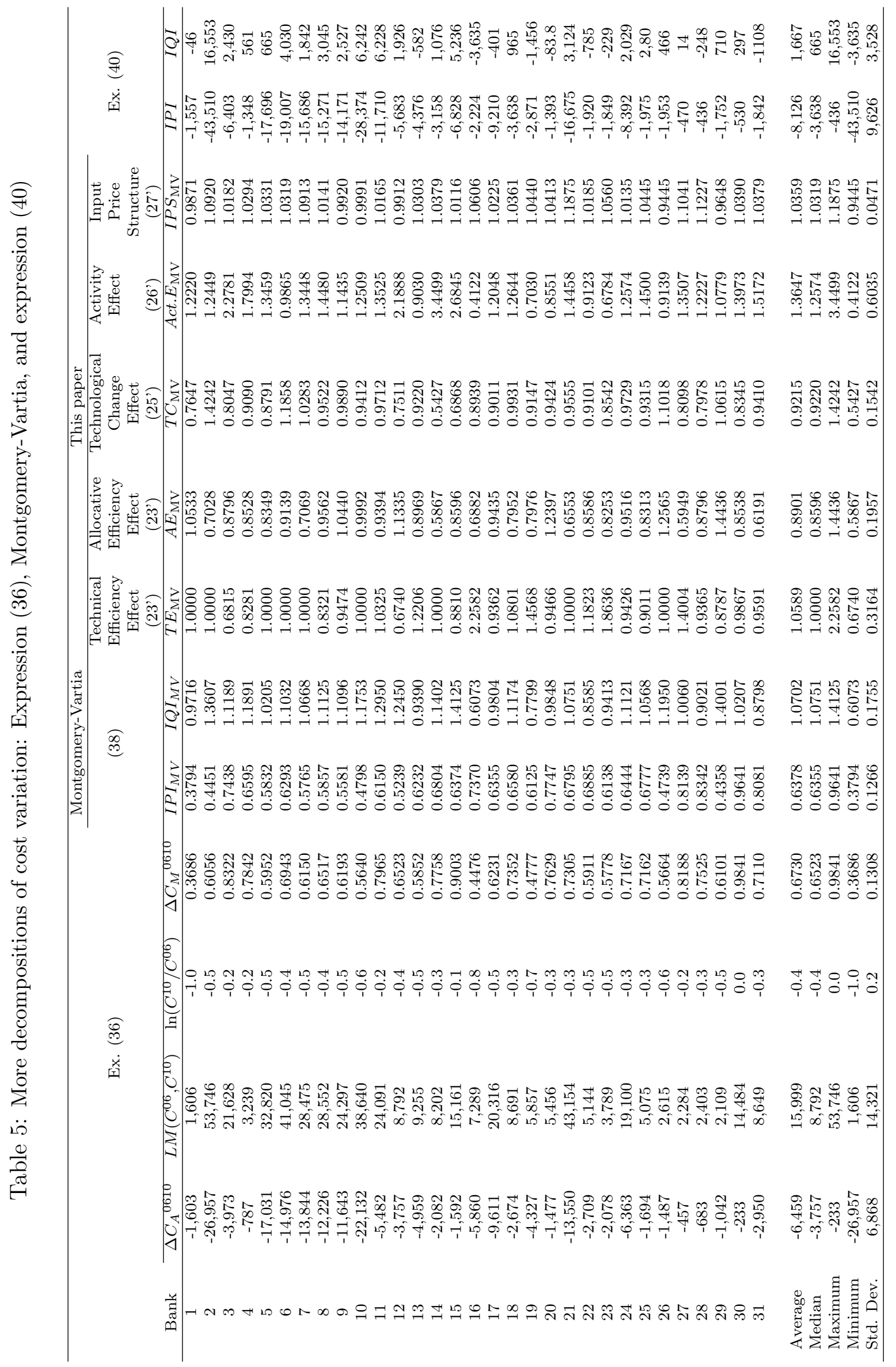

